\title{
A Study to Compare Physiological Cost Index (PCI) of Walking on Treadmill with and without Holding the Hand Rails
}

\author{
Sweety Shah ${ }^{1}$, Ravi Solanki ${ }^{2}$ \\ ${ }^{1}$ SBB Physiotherapy College, AMC MET, Ahmedabad \\ ${ }^{2}$ Government Physiotherapy College, Civil Hospital Campus, Ahmedabad
}

Corresponding Author: Ravi Solanki

\begin{abstract}
Treadmill walking is commonly used for fitness testing and training. Purpose of present study was to determine whether holding the front hand rails makes any significant change in energy expenditure during treadmill walking or not. Thirty volunteers (17 male and 13 female) participated in the study. Subjects were asked to walk on treadmill at speed of 1.0 to $6.0 \mathrm{mph}$ with and without holding the front hand rails for 6 minutes. Physiological cost index was measured for two different positions and compared for statistically significant difference. Results showed that there was no significant difference $(p>0.05)$ in Physiological cost index measured for two different positions. Hence, present study concludes that, holding the front hand rails does not make any significant difference in energy expenditure while walking on treadmill.
\end{abstract}

Keywords: Treadmill walking, Physiological cost index, Hand rail

\section{INTRODUCTION}

Stress testing on treadmill is commonly done to assess the functional capacity, prognosis and diagnosis for patients with cardiopulmonary dysfunctions. Prescription for activities of daily living and intensity for cardiovascular training is based on $\mathrm{VO}_{2}$ max obtained during treadmill test. If measurement of $\mathrm{VO}_{2}$ max is not available then, $\mathrm{VO}_{2}$ max is estimated or predicted usually according to total treadmill time. It has been proven that total treadmill time is markedly longer when subject is allowed to take support of hand rail during treadmill test. ${ }^{[1-4]}$ When $\mathrm{VO}_{2}$ max is predicted from treadmill test duration, it has been found clearly that hand rail support independently overestimates the value of $\mathrm{VO}_{2}$ max. This overestimate of $\mathrm{VO}_{2}$ max increases as total duration test increases. ${ }^{[5]}$ Measured $\mathrm{VO}_{2}$ max has not been found significantly different in tests with or without hand rail support. However, during sub maximal exercise particularly above anaerobic threshold, measured $\mathrm{VO}_{2}$ max is significantly reduced when subject rests his hands or fingers on the front hand rail. ${ }^{[3,4,6 \text {, }}$ 7]

Physiological cost index (PCI) has been developed by MacGregor (1979) for measuring energy expenditure of walking. The PCI has been defined as the difference of walking heart rate and resting heart rate divided by walking speed (that is, $\mathrm{PCI}=$ (walking heart rate- resting heart rate)/walking speed). It is easy to apply in clinics, no heavy equipment is required, and influences of emotional stress, fitness, medication, illness, and ambient temperature are very small. It is concluded that PCI is valid and reliable measure of energy expenditure and has been recommended as a useful tool for physiotherapist in the assessment and evaluation of functional performance. Mean PCI values for males, females and all subjects while walking on treadmill has 
been calculated which were $0.40,0.49$, and 0.44 beats/m respectively.

It has been found that there is no significant difference between the PCI values taken during floor walking and treadmill walking. But Lack of information exists whether holding or not holding the hand rails makes any significant difference in energy expenditure while walking on treadmill. So, purpose of this study is to compare energy expenditure of walking on treadmill with and without holding the hand rails by calculating PCI for both positions.

\section{METHOD}

Thirty male and female subjects ranging in age from 20-25 yrs. volunteered to take part in this study. Subject characteristics are presented in Table 1. The volunteers were healthy, non-smoking, and not taking any medications that would influence the outcome of the study. All subjects signed an informed consent. They were asked to refrain from any activity that was not normally part of their daily routine. All subjects were tested using two protocols. During the first testing session, the subjects were randomly assigned to one of two protocols. The second testing session consisted of the subject performing the protocol that was not completed during the first testing session. Before starting the test, resting HR were measured. First protocol consisted of walking on treadmill with holding the front hand rails for 6 minutes with speed increment of $1.0 \mathrm{mph} /$ minute. At the end of testing, HR (walking) were measured. During second protocol same procedure was repeated except subjects were asked to walk without holding the front hand rails Physiological cost index was calculated for both testing protocols. Between testing protocols, there were at least 24 hrs of separation and all subjects were tested as close to the same time of day as possible for each session. PCI for both protocols were compared using unpaired $t$ test. A probability of $\mathrm{P}<0.05$ was used to determine statistical significance.

\section{RESULT}

A comparison of the effect of walking with handrail support and walking without handrail support is presented in Table 2. Note that the table presents standard deviation, mean difference and $\mathrm{p}$ value. No statistical differences were found in the physiological cost index measured during two different protocols ( $p>0.05$ ).

Table 1. Descriptive data of the subjects.

\begin{tabular}{|l|l|l|l|l|}
\hline $\mathbf{N}$ & Gender & Age (yrs) & Height (in) & Weight (kg) \\
\hline 17 & Male & $24 \pm 2.2$ & $68 \pm 3.1$ & $64 \pm 3.2$ \\
\hline 13 & Female & $22 \pm 2.5$ & $66 \pm 3.3$ & $53 \pm 2.4$ \\
\hline 30 & total & $23 \pm 2.6$ & $67 \pm 3.2$ & $59 \pm 3.1$ \\
\hline
\end{tabular}

Table 2. Comparison of PCI for two different protocols

\begin{tabular}{|l|l|l|l|}
\hline & $\begin{array}{l}\text { With } \\
\text { holding } \\
\text { hand rails }\end{array}$ & $\begin{array}{l}\text { Without } \\
\text { holding hand } \\
\text { rails }\end{array}$ & $p$ value \\
\hline $\begin{array}{l}\text { Physiological cost } \\
\text { index (PCI) }\end{array}$ & $0.27 \pm 0.12$ & $0.3 \pm 0.10$ & 0.226653 \\
\hline
\end{tabular}

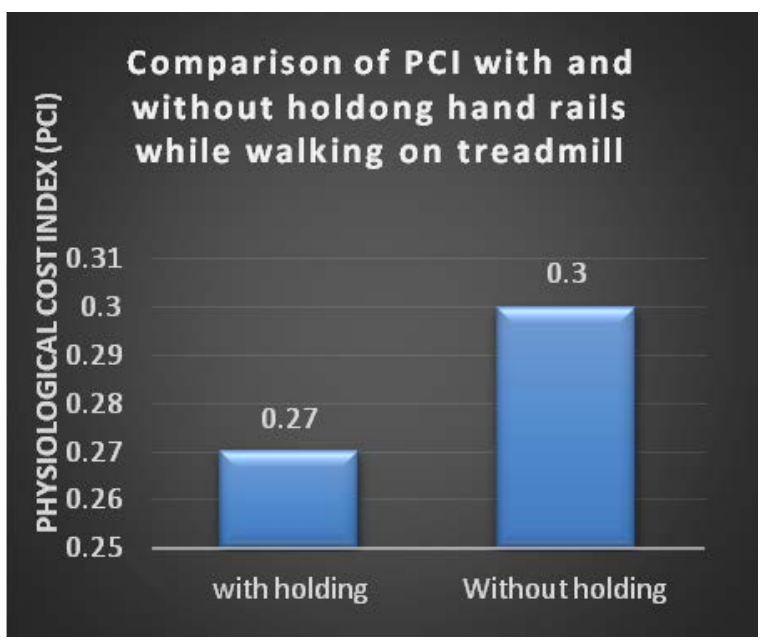

Figure 1: PCI for treadmill walking with and without holding the hand rails

\section{DISCUSSION}

The results of this study did not support the belief that using handrail support during treadmill exercise decreases energy expenditure. Such finding are consistent with the study done by Manfre et al. ${ }^{[8]}$ and, in particular, with Zeimetz et al. ${ }^{[5]}$ who found that VO2 in the initial stages was not changed. Interestingly, Manfre and colleagues ${ }^{[8]}$ analysed total treadmill time between healthy male and female subjects versus male subjects suffering from coronary artery disease and myocardial infarction. The control group was asked to walk with free arm swing and the experimental group was asked to walk with 
handrail support of the tips of two fingers. There was no significant difference in total treadmill time in healthy male subjects. Since total treadmill time was unchanged, it is reasonable to assume that $\mathrm{VO} 2$ did not change either.

The findings of Manfre et al. ${ }^{[8]}$ and the present study contradict the findings in studies that reported a decrease in VO2 when handrail support was utilized. As an example, Christman et al. ${ }^{[9]}$ studied the effects of handrail support while exercising on a step treadmill. There was significant reduction in oxygen consumption and heart rate with light support of hand rail as compared to no support. The significant reduction in HR suggests a reduction in cardiac output that very likely caused the decrease in VO2. The reduction in in Heart rate is also suggestive that myocardial oxygen consumption might have been reduced with support of hand rails.

What is apparent in the present study is this: There are no physiological responses while walking on the treadmill at 1.0-6.0 mph without handrail support that are different from walking on the treadmill at 1.0-6.0 mph while holding onto the handrails. In other words, it does not matter whether the subject holds onto the rails or not. Treadmill walking is commonly prescribed as a part of aerobic training for populations with all age groups. Findings of present study suggest that the use of handrail support while walking on treadmill does not affect the benefits gained from an aerobic exercise program.

\section{CONCLUSION}

Walking with Handrail support on treadmill exercise is not a new concept Many people in a gym setting use the handrails because they feel it allows them to exercise harder and longer, therefore expending more calories. The treadmill machines determine the amount of calories burned is through use of various regression equations. These equations are designed to consider different variables such as grade, speed, weight, height, and heart rate, it does not take in account about hand rail support taken. The results of this study indicate that in normal healthy college-aged adults, PCI was not significantly altered when handrails were used during walking on the treadmill for 6 mins.

\section{ACKNOWLEDGEMENT}

We are thankful to Director and Principal, Govt. Physiotherapy College, Ahmedabad for necessary support in the process of data collection.

\section{Conflict of Interest: None}

\section{Source of Funding: None}

\section{Ethical Approval: Approved}

\section{REFERENCES}

1. Haskell W L, Savin W, Oldridge J, DeBusk $\mathrm{R}$ : Factors influencing estimated oxygen uptake during exercise testing soon after myocardial infarction. Am J Cardiol 1982;50 299-304.

2. Ragg K, Murray T, Karbonit L, Jump D Errors in predicting functional capacity from a treadmill exercise stress test. Am Heart J 1984 ;100(4):581-583.

3. von Duvillard SP, Pivirotto JM: The effect of front handrail and nonhandrail support on treadmill exercise in healthy women. J Cardiopulm Rehab 1991;11:164-168.

4. McConnell TR, Foster C, Conlin N, Thompson N: Prediction of hnctional capacity during treadmill testing: Effect of handrail support. J Cardiopulm Rehab 1991 ;11: 255-260.

5. Zeimetz GA, McNeill .I, Hall J , Moss R: Quantifiable changes in oxygen uptake, heart rate and time to target heart rate when hand support is allowed during treadmill exercise. J Cardiopulm rehab 1985 ;5:525530.

6. Myers J, Buchanan N, Walsh D, Kraemer M, McAuley P, Hmilton- Wessler M, Froelicher V: Comparison ofthe ramp versus standard exercise protocols..J Am Coll Cardiol 1991 17(6), 1334-1342.

7. Sullivan M, McKiman D: Errors in predicting functional capacity for post myocardial infarction patients using a 
Sweety Shah et.al. A study to compare physiological cost index (PCI) of walking on treadmill with and without holding the hand rails.

modified Bruce protocol. Am Heart J 1984;107:486488.

8. Manfre MJ, Yu Guan-Hiok, Varma AA, Mallis GI, Kearney K, Karageorgis MA. The effect of limited handrail support on total treadmill time and the prediction of VO2 max. Clin Cardiol. 1994;17:445-450.

9. Christman SK, Fish AF, Bernhard L, Frid DJ, Smith BA, Mitchell L. Continuous handrail support, oxygen uptake, and heart rate in women during submaximal step treadmill exercise. Res Nurs Health. 2000; 23:35-42.

How to cite this article: Shah S, Solanki R. A study to compare physiological cost index (PCI) of walking on treadmill with and without holding the hand rails. International Journal of Science \& Healthcare Research. 2021; 6(4): 216-219. DOI: https://doi.org/10.52403/ijshr. 20211031 